\title{
Cáncer de recto. Análisis de las complicaciones post operatorias y mortalidad de la resección radical en una serie de 10 años*
}

\author{
Drs. JOSÉ GELLONA V. ${ }^{1}$, FELIPE BELLOLIO R. ${ }^{1}$, M. ELENA MOLINA P. ${ }^{1}$, \\ RODRIGO MIGUIELES C. ${ }^{1}$, GONZALO URREJOLA S.${ }^{1}$, ALVARO ZÚÑIGA D. ${ }^{1}$ \\ 1 Departamento de Cirugía Digestiva. Unidad de Coloproctología. Facultad de Medicina. \\ Pontificia Universidad Católica de Chile. \\ Santiago, Chile.
}

\begin{abstract}
\section{Rectal cancer: early postoperative morbidity and mortality after radical resection}

Introduction: There has been progress in surgical treatment of rectal cancer (CR) in the past decade due to a better knowledge of the disease and the adoption of new methods of preoperative staging and treatment. The aim of this report is to analyze the early postoperative morbidity and mortality in a ten year series of patients with CR submitted to radical resection. Methods: In the database of colorectal cancer patients prospectively maintained, all patients with CR submitted to curative radical resection electively between January 2000 and December 2010 were identified. Early postoperative (30 day) morbidity and mortality were described and analyzed in a multivariate analysis to identify predictive factors. Results: A total of 308 patients were included, 55.2\% male with a median age of 62 years. Over two thirds of tumors were located in the mid or lower rectum. Anterior resection was performed in $83.1 \%$, and neoadyuvant radio-chemotherapy was used in $37.7 \%$. Overall morbidity and mortality were $13.6 \%$ and $0.3 \%$ respectively. In multivariate analysis, American Society of Anesthesiologists had an or of 3.343 (1.601- 6.982) for postoperative morbidity, and laparoscopic approach $0.188(0.054-0.649)$. Conclusion: The morbidity rate of this series is similar to the one observed in other studies. The ASA score is an independent risk factor for postoperative complication and the laparoscopic approach would be a protective factor. In this series, preoperative chemoradiation was not a risk factor for postoperative morbidity.
\end{abstract}

Key words: Rectal, cancer, postoperative complications, predictors.

\section{Resumen}

Introducción: Durante la última década ha habido progresos en el tratamiento del adenocarcinoma del recto (CR) derivado del mejor conocimiento de la enfermedad y de la incorporación de nuevas técnicas de etapificación y tratamiento. El objetivo de este trabajo es analizar la morbilidad y mortalidad en una serie de pacientes sometidos a resección radical (resección anterior o resección abdominoperineal) por CR. Mé-

*Recibido el 1 de octubre de 2012 y aceptado para publicación el 19 de noviembre de 2012.

Los autores no tienen conflictos de interés ni fuentes de financiamiento que reportar.

Correspondencia: Dr. José Gellona V.

Marcoleta 350 patio interior, Santiago, Chile.

jgellona@yahoo.com 
todo: Estudio de cohorte histórica. Se identificaron los pacientes operados por CR, en forma electiva y con intención curativa entre enero de 2000 y diciembre de 2010. Se analizó la morbilidad global y mortalidad a 30 días. Además se realizó un análisis uni y multivariado para encontrar factores predictores de complicaciones. Resultados: La serie consta de 308 pacientes, $55,2 \%$ de sexo masculino con una mediana de edad de 62 años. Se observó una tasa de morbilidad global de 13,6\%. Las complicaciones más frecuentes fueron el íleo prolongado $(3,2 \%)$, filtración de anastomosis $(2,3 \%)$ e infección de sitio quirúrgico $(1,3 \%)$. La mortalidad operatoria fue de $0,3 \%$. En el análisis multivariado, el puntaje ASA se asoció significativamente a morbilidad. El abordaje laparoscópico se asoció a una menor tasa de complicaciones. No se observó asociación con neoadyuvancia ni tipo de cirugía. Conclusión: En esta serie, la tasa de complicaciones es similar a lo comunicado en otras series. El puntaje ASA es un factor de riesgo independiente para complicación y, por el contrario, el abordaje laparoscópico sería un factor protector. La radioquimioterapia neoadyuvante no sería, según esta serie, un factor de morbilidad.

Palabras clave: Cáncer, recto, complicaciones, predictores.

\section{Introducción}

En Chile, la mortalidad por cáncer colorrectal ha mostrado un progresivo aumento en los últimos 20 años $^{1}$. Los adenocarcinomas de recto (CR) corresponden aproximadamente al $30 \%$ de todas las neoplasias colorrectales. El pilar fundamental del tratamiento es la cirugía, que se ve dificultada por la localización anatómica del recto, profundo entre las paredes rígidas de la pelvis, y el deseo de conservar el aparato esfinteriano. Los objetivos centrales del tratamiento quirúrgico radical (resección anterior y resección abdominoperineal) son la extirpación total del tumor y su territorio linfático ${ }^{2,3}$, evitar la recurrencia local y mantener una adecuada calidad de vida. Sin embargo, para lograr estos objetivos, los pacientes se exponen a complicaciones postoperatorias que pueden derivar en hospitalizaciones prolongadas, necesidad de cuidados especiales, mortalidad operatoria y compromiso de los resultados funcionales ${ }^{4}$.

A lo largo de la última década, el mejor conocimiento de la enfermedad, la incorporación de nuevas técnicas de estadificación ${ }^{5-8}$, el empleo de la neoadyuvancia ${ }^{9-12}$ y la introducción de la resección por videolaparoscopía ${ }^{13}$, han sido importantes avances que podrían afectar los resultados quirúrgicos. En la literatura nacional, existen publicaciones al respecto $^{14-16}$, pero de subgrupos de pacientes con tumores del tercio superior del recto ${ }^{14}$, o sometidos a resección abdominoperineal incluyendo pacientes con cáncer ana ${ }^{16}$. En este escenario parece necesario revisar los resultados inmediatos del tratamiento quirúrgico radical del CR en términos de morbilidad y mortalidad.

El objetivo de este estudio es analizar las complicaciones del tratamiento quirúrgico del CR y tratar de identificar factores predictores de morbilidad, en una serie de pacientes operados durante la última década, donde se han incorporado las innovaciones mencionadas.

\section{Material y Método}

En la base de datos de neoplasias colorrectales mantenida en forma prospectiva, se identificaron todos los pacientes sometidos a resección radical (resección anterior o resección abdominoperineal), electiva y con intención curativa por $\mathrm{CR}$, en el período entre enero de 2000 y diciembre de 2010. Se consideró como CR los adenocarcinomas a menos de $15 \mathrm{~cm}$ del margen anal que se dividieron en tercio inferior $(0-7 \mathrm{~cm})$, medio $(8-11 \mathrm{~cm})$ y superior $(12-15 \mathrm{~cm})$.

El estudio preoperatorio incluyó hemograma, perfil bioquímico, antígeno carcinoembrionario, colonoscopía, biopsia, tomografías de tórax, abdomen y pelvis, resonancia magnética de pelvis y/o endosonografía rectal. Todos los pacientes se evaluaron en comité oncológico multidisciplinario. Los pacientes con tumores de tercio medio o inferior localmente avanzados (T3 y T4), se sometieron a radio - quimioterapia preoperatoria de curso largo (45 Gy a la pelvis con refuerzo de 5 Gy al tumor, asociado a 5-FU en un período de 6 semanas). Además, algunos tumores avanzados en el tercio superior (T3 voluminosos o T4), también recibieron radio-quimioterapia. Los pacientes que recibieron neoadyuvancia, fueron operados entre 6 a 8 semanas después de la última dosis de radioterapia. Todos fueron operados por cirujanos colorrectales o residentes de la especialidad bajo supervisión directa. La técnica quirúrgica, independiente del abordaje (abierto o laparoscópico), incluyó la resección total del mesorrecto y ligadura de los vasos mesentéricos en su origen. En los casos de resección anterior, la anastomosis colorrectal se practicó con doble grapadura. En algunos casos de resecciones anteriores bajas se realizó anastomosis coloanal manual por vía anal. El uso de ileostomía de protección se indicó según criterio del cirujano. En todos los pacientes se dejó un drenaje tubular en la pelvis extraído por contrabertura en la pared abdominal. El estudio anátomo patológico de la 
pieza operatoria se realizó en base a los criterios de la clasificación TNM.

Se registraron datos demográficos, variables del tumor, uso de neoadyuvancia, operación practicada, abordaje quirúrgico (laparoscópico y clásico), complicaciones postoperatorias y mortalidad a 30 días. Se consideró como filtración de anastomosis la comprobación de extravasación de líquido intestinal en el drenaje, imágenes o endoscopía. No se practicó estudio imagenológico para detección de filtración en los pacientes asintomáticos. Las siguientes variables se estudiaron como factores de riesgo para complicación: sexo, edad, ASA, uso de neoadyuvancia, localización del tumor, operación practicada, abordaje y etapificación final. La variable edad, además de analizarla como variable continua, se categorizó arbitrariamente en 2 grupos, menores y mayores de 80 años, dado que se estimó que los pacientes sobre los 80 años podrían tener mayor riesgo quirúrgico. Para determinar la gravedad de las complicaciones se utilizó la clasificación de Clavien y Dindo ${ }^{17}$.

Para el estudio de asociación univariado entre variables se utilizaron tablas de asociación $\left(\chi^{2}\right.$ y prueba exacta de Fisher según corresponda). Para el análisis multivariado se utilizó regresión logística binaria. Se incorporaron las variables que se estimó que podrían afectar los resultados quirúrgicos a corto plazo como son: el uso de neoadyuvancia y el abordaje laparoscópico. Además se incorporó a dicho análisis cualquier variable que aunque no fuese significativa en el análisis univariado, la magnitud de la diferencia mostrara una tendencia clara. Para el análisis estadístico se utilizó STATA $10^{\mathrm{R}}$, y para efectos de significación estadística se fijó un alfa menor a 0,05 .

El objetivo primario es mostrar la morbilidad y mortalidad operatoria. El objetivo secundario es determinar si entre los factores analizados, existen predictores de morbilidad.

\section{Resultados}

En el período mencionado fueron operados 361 pacientes con CR, de los cuales, 308 cumplían los criterios de inclusión y forman la serie analizada. Las características generales de la serie se muestran en la Tabla 1. Se observó que un poco más de la mitad de los enfermos eran de sexo masculino $(55,2 \%)$, la mayoría $(75 \%)$ con patologías crónicas compensadas (ASA 2). El mayor porcentaje de los tumores $(72 \%)$ se ubicaron en el tercio medio e inferior y $24 \%$ tercio superior. Más de un tercio $(37,7 \%)$ recibió neoadyuvancia. La operación realizada con mayor frecuencia fue la resección anterior $(83,1 \%)$, con ileostomía de protección en 57,5\% de ellos. En
4 pacientes se realizó la resección anterior de recto tipo Hartmann. Un 29,2\% de los pacientes se abordaron por laparoscopía, con 8 (8,9\%) conversiones. Según el estudio anátomo - patológico, 23 pacientes (19,8\% de los pacientes con neoadyuvancia) fueron etapa 0 (respuesta patológica completa); $69(22,4 \%)$ etapa I; $101(32,8 \%)$ etapa II, $84(27,2 \%)$ etapa III y $21(6,8 \%)$ etapa IV. En $10(3,2 \%)$ pacientes no se dispuso del estudio histológico postoperatorio.

Una o más complicaciones post operatorias se observaron en 42 pacientes (morbilidad global: $13,6 \%$ ). El detalle de las complicaciones se muestra en la Tabla 2. La complicación más frecuente fue el íleo prolongado (no reinicio del tránsito intestinal a gases o deposiciones en 7 días), que se observó en $10(3,2 \%)$ pacientes. Todos fueron manejados en forma conservadora con resolución del cuadro. Filtración de la anastomosis se observó en 6 pacientes $(2,3 \%$ de los pacientes con anastomosis). Cuatro de ellos que tenían ileostomía de protección y drenaje cuáles fueron manejados con antibióticos. Dos pacientes debieron ser reintervenidos. En uno se practicó aseo, drenaje e ileostomía en asa, y en el otro colostomía y cierre del muñón rectal. Un paciente $(0,3 \%)$, abordado por vía laparoscópica, presentó necrosis del colon descendido a la pelvis.

Tabla 1. Características generales de la serie

\begin{tabular}{|c|c|}
\hline Variable & n $\quad(\%)$ \\
\hline Total pacientes & 308 \\
\hline Sexo (masculino) & $170(55,2)$ \\
\hline Edad & $62(22-96)^{*}$ \\
\hline \multicolumn{2}{|l|}{ ASA } \\
\hline 1 & $85(27,6)$ \\
\hline 2 & $146(47,4)$ \\
\hline 3 & $19(6,2)$ \\
\hline Sin información & $64(20,8)$ \\
\hline \multicolumn{2}{|l|}{ Localización del tumor rectal } \\
\hline Superior & $74(24)$ \\
\hline Medio o inferior & $222(72,1)$ \\
\hline Sin información & $12(3,9)$ \\
\hline Neoadyuvancia & $116(37,7)$ \\
\hline \multicolumn{2}{|l|}{ Operación } \\
\hline Resección anterior & $256(83,1)$ \\
\hline Resección abdominoperineal & $44(14,3)$ \\
\hline Otras** & $8(2,6)$ \\
\hline Ileostomía de protección & $177(57,5)$ \\
\hline Abordaje laparoscópico & $90(29,2)$ \\
\hline
\end{tabular}

*Resultado expresado en mediana (mínimo-máximo). **Proctocolectomías (4), resecciones tipo Hartmann (4). 
Tabla 2. Complicaciones post operatorias $(n=308)$

\begin{tabular}{|lrc|}
\hline Complicación & $\mathbf{n}$ & $\mathbf{\%}$ \\
Íleo postoperatorio & & \\
Filtración anastomosis & 10 & 3,2 \\
Respiratorias & 6 & $2,3 *$ \\
Infección sitio quirúrgico & 5 & 1,6 \\
Evisceración & 4 & 1,3 \\
Hemorragia digestiva & 3 & 1,0 \\
Colección & 2 & 0,6 \\
Hemoperitoneo & 2 & 0,6 \\
Complicaciones enterostomía & 2 & 0,6 \\
Necrosis / isquemia (colon descendido) & 1 & 0,3 \\
Perforación & 1 & 0,3 \\
Obstrucción intestinal & 1 & 0,3 \\
Accidente vascular cerebral & 1 & 0,3 \\
Síndrome coronario agudo & 1 & 0,3 \\
Insuficiencia renal aguda & 1 & 0,3 \\
Trombosis venosa profunda & 1 & 0,3 \\
Total & 1 & 0,3 \\
Gravedad de la complicación** & 42 & 13,6 \\
I & & \\
II & 7 & 2,3 \\
IIIa & 3 & 1,3 \\
IVa & 1 & 0,3 \\
V & & 2,3 \\
\hline
\end{tabular}

*Porcentaje de los pacientes con anastomosis de la serie. **Según clasificación de Clavien y Dindo ${ }^{17}$.

Fue reoperado con resección del colon isquémico. Un paciente que había sido abordado por laparotomía evolucionó con peritonitis por perforación inadvertida de colon. Fue operado precozmente con buena evolución posterior. Las otras complicaciones que fueron manejadas quirúrgicamente fueron: 2 $(0,6 \%)$ evisceraciones, $2(0,6 \%)$ hemoperitoneos y una nueva confección de ostomía por necrosis. En total, $8(2,6 \%)$ pacientes necesitaron ser reintervenidos. Se observó además un accidente vascular cerebral isquémico sin secuelas, un síndrome coronario agudo, una trombosis venosa profunda y una insuficiencia renal aguda que no requirió diálisis. Un paciente de la serie falleció (mortalidad operatoria $0,3 \%$ ). Había sido sometido a una resección anterior por vía laparoscópica y evolucionó con un infarto masivo postoperatorio.

En el análisis univariado para morbilidad general,
Tabla 3. Análisis univariado de factores de riesgo para morbilidad general

\begin{tabular}{|c|c|c|c|}
\hline Variable & $\begin{array}{c}\text { Sin } \\
\text { complicación } \\
\text { n }(\%)\end{array}$ & $\begin{array}{c}\text { Con } \\
\text { complicación } \\
\text { n }(\%)\end{array}$ & p \\
\hline \multicolumn{4}{|l|}{ Sexo } \\
\hline Femenino & $124(89,9)$ & $14(10,1)$ & NS \\
\hline Masculino & $142(83,5)$ & $28(16,5)$ & \\
\hline Edad & $62 \pm 19$ & $66,5 \pm 22$ & NS \\
\hline $\begin{array}{l}\text { Edad } \\
\text { Menor a } 80 \text { años } \\
\text { Mayor a } 80 \text { años }\end{array}$ & $\begin{array}{r}237(85,3) \\
29(96,7)\end{array}$ & $\begin{array}{r}41(14,7) \\
1(3,3)\end{array}$ & NS \\
\hline $\begin{array}{r}\text { ASA } \\
1-2 \\
3-4\end{array}$ & $\begin{array}{r}179(86,5) \\
12(70,6)\end{array}$ & $\begin{array}{r}28(13,5) \\
5(29,4)\end{array}$ & NS \\
\hline $\begin{array}{l}\text { Localización } \\
\text { Superior } \\
\text { Medio o inferior }\end{array}$ & $\begin{array}{r}59(79,7) \\
195(87,4)\end{array}$ & $\begin{array}{l}15(20,3) \\
27(12,2)\end{array}$ & NS \\
\hline $\begin{array}{l}\text { Cirugía } \\
\text { R. anterior } \\
\text { RAP } \\
\text { Otra }\end{array}$ & $\begin{array}{c}221(86,3) \\
39(88,6) \\
6(75)\end{array}$ & $\begin{aligned} 35 & (13,7) \\
5 & (11,4) \\
2 & (25)\end{aligned}$ & NS \\
\hline $\begin{array}{l}\text { Ileostomía } \\
\text { No } \\
\text { Sí }\end{array}$ & $\begin{array}{l}114(87) \\
152(85,9)\end{array}$ & $\begin{array}{c}17(13) \\
25(14,1)\end{array}$ & NS \\
\hline $\begin{array}{l}\text { Etapa } \\
0 \text { - II } \\
\text { III - IV }\end{array}$ & $\begin{array}{r}159(87,9) \\
85(80,8)\end{array}$ & $\begin{array}{l}21(11,7) \\
19(18,3)\end{array}$ & NS \\
\hline $\begin{array}{l}\text { Neoadyuvancia } \\
\text { No } \\
\text { Sí }\end{array}$ & $\begin{array}{l}165(85,9) \\
101(87,1)\end{array}$ & $\begin{array}{l}27(14,1) \\
15(12,9)\end{array}$ & NS \\
\hline $\begin{array}{l}\text { Laparoscopía } \\
\text { No } \\
\text { Sí }\end{array}$ & $\begin{array}{r}180(82,6) \\
86(95,6)\end{array}$ & $\begin{array}{r}38(17,4) \\
4(4,4)\end{array}$ & 0,002 \\
\hline
\end{tabular}

RAP: Resección abdomino perineal.

el único factor asociado fue el abordaje laparoscópico, que resultó un factor protector, con un OR de 0,22 con un IC $95 \%$ entre 0,076 y 0,637 . No se observaron otras asociaciones significativas al analizar operación practicada, localización de la lesión y radio - quimioterapia preoperatoria (Tabla 3 ). Al realizar el análisis multivariado, ajustando por sexo, laparoscopía, edad, ASA, localización tumoral y neoadyuvancia, encontramos que la asociación protectora entre laparoscopía y complicación general se mantiene; al ajustar por los otros factores incorporados al modelo, se agrega como factor de riesgo el puntaje ASA, con un OR de 3,34 (Tabla 4). 
Tabla 4. Análisis multivariado para morbilidad global

\begin{tabular}{|lccc|}
\hline Variable & $\begin{array}{l}\text { Odds } \\
\text { ratio }\end{array}$ & $\mathbf{9 5 \%}$ IC & p \\
\hline Laparoscopía & 0,155 & $0,043-0,556$ & 0,004 \\
\hline $\begin{array}{l}\text { ASA (III-IV) } \\
\text { Neoadyuvancia }\end{array}$ & 3,343 & $1,601-6,982$ & 0,001 \\
\hline $\begin{array}{l}\text { Localización (recto } \\
\text { medio o inferior } \\
\text { versus superior) }\end{array}$ & 0,654 & $0,288-1,485$ & NS \\
\hline $\begin{array}{l}\text { Edad (mayor a } 80 \text { años) } \\
\text { Sexo masculino }\end{array}$ & 0,196 & $0,021-1,788$ & NS \\
\hline
\end{tabular}

\section{Discusión}

Los resultados observados en este estudio muestran una morbilidad global de $13,6 \%$ y una mortalidad de $0,3 \%$ en pacientes sometidos a cirugía radical electiva curativa por CR. El puntaje ASA fue el único predictor de complicaciones y el abordaje laparoscópico sería un factor protector. Estos resultados se comparan favorablemente con otras series que comunican la morbilidad en pacientes con CR operado. Paun y cols, en un meta análisis que incluyó sobre 36.000 pacientes en todos los escenarios clínicos, comunicaron una tasa de filtración de anastomosis del $11 \%$, sepsis pélvica de $12 \%$, infección de sitio quirúrgico $7 \%$ y una mortalidad del $2 \%{ }^{4}$. Los resultados de esta serie son favorables posiblemente debido a la exclusión de pacientes operados de urgencia, a la estricta evaluación preoperatoria de las comorbilidades y también porque en nuestra institución, todos los procedimientos son realizados o supervisados directamente por un cirujano colorrectal con una técnica quirúrgica uniforme.

Por otra parte, nuestros resultados son consistentes en asociar el puntaje ASA como factor de riesgo para complicación. Esto concuerda con otras series que comunican las complicaciones luego de la cirugía por $\mathrm{CR}^{18,19}$.

El abordaje laparoscópico en esta serie resultó un factor protector, mostrando un Odds Ratio de 0,155 , es decir, 6 veces menos probabilidad de sufrir una complicación. Esto se podría explicar en parte por un sesgo de selección, ya que en el inicio de la experiencia laparoscópica, se seleccionaron los pacientes más favorables para esta técnica: tumores de menor tamaño y pacientes delgados sin patologías asociadas. Una vez consolidada la experiencia laparoscópica se comenzaron a operar pacientes con comorbilidades cardíacas y/o pulmonares en las cuales la técnica laparoscópica tiene menor impacto en el manejo postoperatorio. Estos resultados son compatibles con lo comunicado en grupos con experiencia, donde el abordaje laparoscópico disminuye la morbilidad ${ }^{20-22}$.

Pese a que en este análisis no encontramos otras asociaciones significativas, se han descrito asociaciones con variables estudiados en este trabajo. El sexo masculino por ejemplo, se ha asociado a complicación general. Así, Bennis et $\mathrm{al}^{23}$, en pacientes con resección anterior baja y anastomosis coloanal, observaron que los pacientes de sexo masculino poseían un OR de 1,8 para morbilidad global y 2,3 para complicación médica. Por su parte, Ptok et $\mathrm{al}^{24}$, al analizar, sexo como factor de riesgo para filtración de anastomosis, encontraron que los pacientes de sexo masculino tenían una tasa de filtración más alta. Otros autores han encontrado resultados simila$\operatorname{res}^{25-27}$. Este aumento en el riesgo de complicaciones sería explicado por la estrechez de la pelvis masculina, que confiere mayor dificultad técnica.

La etapa avanzada también se ha asociado a mayor tasa de complicaciones en varias series ${ }^{28}$, aunque con resultados dispares ${ }^{29-32}$.

La localización tumoral también se ha descrito como factor de riesgo para complicaciones, esto es, a menor distancia del margen anal, mayores tasas de complicaciones. En esta serie, no se observó lo anteriormente expuesto. En el análisis univariado hubo un mayor porcentaje de complicaciones en el tercio superior del recto, que no fue estadísticamente significativo. Al incorporar esta variable al análisis multivariado ajustado por neoadyuvancia, ASA, edad, laparoscopía y sexo, tampoco resultó un factor asociado a complicaciones postoperatorias.

Otros factores de riesgo se han mencionado en la literatura para predecir complicaciones en cirugía de CR. Entre ellos, las pérdidas de volumen sanguíneo, necesidad de transfusión, tiempo quirúrgico, uso de ileostomía, uso de drenajes, obesidad, tabaco, edad, drenaje transrectal, etc. ${ }^{18-21,23-25}$. Entre estos, el score de ASA es uno de los factores que se menciona sistemáticamente como factor de riesgo ${ }^{18,19}$ lo que es concordante con lo observado en este estudio.

En esta serie se aprecia que la radio - quimioterapia preoperatoria no es un factor de riesgo de morbilidad postoperatoria. En el análisis de este hallazgo habría que considerar que la mayoría de los pacientes que se sometieron a neoadyuvancia corresponden a tumores del tercio medio o inferior. $\mathrm{Al}$ incluir en este estudio tumores de los 3 tercios podrían verse afectados favorablemente los resultados de morbilidad. Sin embargo, hecho el ajuste excluyendo los tumores de tercio superior los resultados no se vieron afectados, apoyando la robustez de los resultados.

La neoadyuvancia y el abordaje laparoscópico se 
quisieron analizar porque son, en efecto, conductas que se agregaron progresivamente a lo largo de los 10 años de esta serie. El que no hayan sido factores predictores de complicación apoya la teoría de que al incorporar un cambio en la conducta terapéutica, esta no debería afectar la morbilidad si se realiza gradual y selectivamente en grupos quirúrgicos cohesionados que comparten criterios comunes y con apoyo multidisciplinario.

Este estudio tiene ciertas limitaciones. El análisis retrospectivo de los datos, que hace que los resultados de causalidad deban ser interpretados con precaución. La pérdida de datos, es otro problema que podría limitar la interpretación de los resultados. No fue posible analizar variables importantes como la necesidad de transfusión y el IMC preoperatorio, lo que también debe considerarse una limitación de este trabajo.

En conclusión, se puede afirmar que en esta serie de 10 años de tratamiento quirúrgico radical del cáncer de recto, donde se han incorporado nuevas modalidades terapéuticas, las tasas de morbilidad y mortalidad se comparan favorablemente con otras series similares. La incorporación de nuevos recursos terapéuticos (laparoscopía, neoadyuvancia) no se asociaron a mayor tasa de complicaciones y el único factor predictor de complicación significativo fue el puntaje ASA.

\section{Referencias}

1. Donoso A, Villarroel L, Pinedo G. Aumento de la mortalidad por cáncer de colon en Chile, 1990-2003. Rev Med Chile 2006;134:152-8.

2. Heald RJ. A new approach to rectal cancer. Br J Hosp Med. 1979;22:277-81.

3. Heald RJ, Husband EM, Ryall RD. The mesorrectum in rectal cancer surgery the clue to pelvic recurrence? $\mathrm{Br} \mathrm{J}$ Surg. 1982;69:613-6.

4. Paun B, Cassie S, MacLean A, Dixon E, Bauie WD. Postoperative complications following surgery for rectal cancer. Ann Surg. 2010;251:807-18.

5. Hodgman CG, MacCarty RL, Wolff BG, May GR, Berquist TH, Sheedy PF 2nd, et al. Preoperative staging of rectal carcinoma by computed tomography and $0.15 \mathrm{~T}$ magnetic resonance imaging. Preliminary report. Dis Colon Rectum 1986;29:446-50.

6. Butch RJ, Stark DD, Wittenberg J, Tepper JE, Saini S, Simeone JF, et al. Staging of rectal cancer by MR and CT. AJR Am J Roentgenol. 1986;146:1155-60.

7. Hildebrandt U, Feifel G. Endorectal sonography. Surg Annu. 1990;22:169-83.

8. Beynon J, Mortensen NJ, Foy DM, Channer JL, Rigby $\mathrm{H}$, Virjee J. Preoperative assessment of mesorectal lymph node involvement in rectal cancer. Br J Surg.
1989;76:276-9.

9. Camma C, Giunta M, Fiorica F, Pagliaro L, Craxì A, Cottone M. Preoperative radiotherapy for resectable rectal cancer: a meta-analysis. JAMA 2000;284:100815.

10. Gray R, Hills R, Stowe R, Birmingham M, Clarke M, Peto R, et al. Adjuvant radiotherapy for rectal cancer: a systematic overview of 8,507 patients from 22 randomised trials. Lancet 2001;358:1291-304.

11. Glimelius B, Gronberg H, Jarhult J, Wallgren A, Cavallin-Stahl E. A systematic overview of radiation therapy in rectal cancer. Acta Oncol. 2003;42:476-92.

12. Sauer R, Fietkau R, Wittekind C, Martus P, Rödel C, Hoenberger W, et al. Adjuvant versus neoadjuvant radiochemotherapy for locally advanced rectal cancer. Strahlenther Onkol. 2001;177:173-81.

13. Jacobs M, Verdeja JC, Goldstein HS. Minimally invasive colon resection (laparoscopic colectomy). Surg Laparosc Endosc. 1991;1:144-50.

14. Bannura G, Cumsille G, Barrera A, Contreras J, Melo C, Soto D. Resultados del tratamiento quirúrgico del cáncer del tercio superior del recto y de colon sigmoides: Análisis comparativo. Rev Chil Cir. 2009;61:538-43.

15. Suárez J, González P, Giannini $\mathrm{O}$, Rey G, González R, Adelsdorfer C, y cols. Buen control local y sobrevida post resección curativa de cáncer de recto medio e inferior. Protocolo terapéutico multimodal, selectivo. Rev Chil Cir. 2003;55:584-90.

16. Cárdenas R, Pizarro P. Resección abdominoperineal del recto. Análisis de la morbimortalidad operatoria. Rev Chil Cir. 2002;54:21-6.

17. Dindo D, Demartines N, Clavien PA. Classification of Surgical Complications. A New Proposal with Evaluation in a Cohort of 6336 Patients and Results of a Survey. Ann Surg. 2004;240:205-13.

18. Warschkow R, Steffen T, Thierbach J, Bruckner T, Lange J, Tarantino I. Risk factors for anastomotic leakage after rectal cancer resection and reconstruction with colorectostomy. A retrospective study with bootstrap analysis. Ann Surg Oncol. 2011;10:2772-82.

19. Xiao L, Zhang WB, Jiang PC, Bu XF, Yan Q, Li H, et al. Can transanal tube placement after anterior resection for rectal carcinoma reduce anastomotic leakage rate? A single institution prospective randomized study. World J Surg. 2011;35:1367-77.

20. Mackela JT, Kiviniemi H, Laitinen S. Risk factors for anastomotic leakage after left-sided colorectal resection with rectal anastomosis. Dis Colon Rectum 2003;46:653-60.

21. Lujan J, Valero G, Biondo S, Espin E, Parrilla P, Ortiz H. Laparoscopic versus open surgery for rectal cancer: results of a prospective multicentre analysis of 4,970 patients. Surg Endosc. 2012 Jun 27. [Epub ahead of print]. Citado el 8 de agosto de 2012. Disponible en www.springerlink.com/content/100368/

22. Guillou P, Quirke P, Thorpe H, Walker J, Jayne D.G, 
Smith A, et al. Short-term endpoints of conventional versus laparoscopic assisted surgery in patients with colorectal cancer (MRC CLASICC trial): multicentre, randomised controlled trial. Lancet 2005;365:1718-26.

23. Bennis M, Park Y, Lefevre j, Chafai N, Atal E, Tiret E. Morbidity risk factors after total mesorectal excision and coloanal anastomosis. Ann Surg. 2012;255:504-10.

24. Ptok H, Marusch F, Meyer F, Schubert D, Gastinger I, Lippert H. Impact of anastomotic leakage on oncological outcome after rectal cancer resection. Br J Surg. 2007;94:1548-54.

25. Alves A, Panis Y, Mathieu P, Kwiatkowski F, Slim F, Mantion G, et al. Mortality and morbidity after surgery of mid and low rectal cancer. Results of a French prospective multicentric study. Gastroenterol Clin Biol. 2005;29:509-14.

26. Stelzmueller I, Zitt M, Aigner F, Kafka-Ritsch R, Jager $\mathrm{R}$, De Varies A, et al. Postoperative morbidity following chemoradiation for locally advanced low rectal cancer. J Gastrointest Surg. 2009;13:657-67.

27. Peeters KC, Tolennaar RA, Marijnen CA, Klein E, Steup WH, Wiggers T, et al. Risk factors for anasto- motic failure after total mesorectal excision of rectal cancer. Br J Surg. 2005;92:211-6.

28. Lujan J, Valero G, Hernández Q, Sánchez Q, Frutos MD, Parrilla P. Randomized clinical trial comparing laparoscopic and open surgery in patient with rectal cancer. Br J Surg. 2009;96:982-9.

29. Law WI, Chu KW, Ho JW, Chan CW. Risk factors for anastomotic leakage after low anterior resection with total mesorectal excision. Am J Surg. 2000;179:92-6.

30. Baik SH, Gincherman M, Mutch MG, Birnbaum EH, Fleshmann JW. Laparoscopic vs open resection for patients with rectal cancer: comparison of perioperative outcomes and long term survival. Dis Col Rectum 2011;54:6-14.

31. Breunink S, Pierie JP, Wiggers T. Laparoscopic versus open total mesorectal excision for rectal cancer; Cochrane Database of Sistematic Reviews 2008; 4. Citado el 7 de julio de 2012. Disponible en www.cochrane.org.

32. Taflampas P, Christodoulakis M, Tsistis D. Anastomotic leakage after low anterior resection for rectal cancer: Facts, obscurity and fiction. Surgery Today 2009;39:133-88. 